UDC 1(091):130.2

LBC 87.3:71.0

\title{
WASSILY KANDINSKY AND ARNOLD SCHOENBERG: THE ANTICIPATION OF POSTMODERNITY IN ARTS
}

\author{
Aleksandr I. Pigalev \\ Volgograd State University, Volgograd, Russian Federation
}

\begin{abstract}
The aim of the article is a comparative analysis of the principles of abstract painting and atonal music as an anticipation of postmodernity in arts, which is understood as an appropriate response to the severe crisis of modernity. It is indicated that the striking resemblance of the ideas of Wassily Kandinsky and Arnold Schoenberg was caused by structural changes in the European metaphysics of subjectivity, the cause of which was the approaching postmodernity. The transition from modernity to postmodernity is seen as the destruction of all centered and closed integrity which followed the model of metaphysics, and thereupon required invention of new types of ordering. It is emphasized that atonal music is based on the assumption of the absence of tonic and equality of twelve tones of chromatic scale organized in the series. As a result, its structure reproduces the outcome of the destruction of metaphysics at another level of culture and through dissimilar symbols. The rejection of the norms of linear perspective as the basis of representationalism in painting and the subsequent denial of the principles of compatibility and order of colors in Kandinsky's concept of abstract art of means the destruction of the same deep structure. This destruction affected, first of all, the position of the self-asserting subject as the pinnacle of the metaphysics of modernity, and also caused the failure of mechanisms generating and maintaining the confidence of modernity in its universality and eternity, which seemed to follow from its unique metaphysical foundations.
\end{abstract}

Key words: Wassily Kandinsky, Arnold Schoenberg, postmodernity, metaphysics, tonality, atonality.

УДК 1(091):130.2

ББК 87.3:71.0

\section{ВАСИЛИЙ КАНДИНСКИЙ И АРНОЛЬД ШЁНБЕРГ: ПРЕДВОСХИЩЕНИЕ ПОСТМОДЕРНА В ИСКУССТВЕ}

\author{
Александр Иванович Пигалев \\ Волгоградский государственный университет, г. Волгоград, Российская Федерация
}

\begin{abstract}
Аннотация. Целью статьи является сравнительный анализ принципов абстрактной живописи и атональной музыки в качестве предвосхищения постмодерна в искусстве, которое понимается как закономерная реакция на глубокий кризис модерна. Указывается, что поразительное сходство идей Василия Кандинского и Арнольда Шёнберга было вызвано структурными изменениями европейской метафизики субъективности, причиной которых стал приближающийся постмодерн. Переход от модерна к постмодерну рассматривается как разрушение всех центрированных и замкнутых целостностей, которые следовали образцу метафизики, а затем испытывали потребность в создании новых типов упорядочения. Подчеркивается, что атональная музыка основана на допущении отсутствия тоники и равенства двенадцати тонов хроматической гаммы, которые организованы в серии. В результате ее структура воспроизводит исход разрушения метафизики на $\underset{0}{\infty}$ другом уровне культуры и посредством несходных символов. Отказ от норм линейной перспективы в качеح стве основания репрезентационизма в живописи и последующее отрицание принципов сочетаемости и порядка цветов в концепции абстрактного искусства Кандинского означает разрушение той же глубинной структуры. Это разрушение коснулось в первую очередь положения самоутверждающегося субъекта в качестве вершины метафизики модерна, а также вызвало сбой механизмов, порождающих и поддерживающих уверенность модерна в своей универсальности и вечности, которые, как казалось, следуют из его единственных в своем роде метафизических оснований.
\end{abstract}


Ключевые слова: Василий Кандинский, Арнольд Шёнберг, постмодерн, метафизика, тональность, атональность.

Известно, что русский художник Василий Васильевич Кандинский (1866-1944) и австрийский композитор Арнольд Шёнберг (1874-1951) в переписке и теоретических работах подчеркивали идейную близость друг другу, и именно в этом общем контексте обычно рассматривается их совместное влияние на искусство и эстетику модернизма. Важным итогом такого рассмотрения стало доказательство единства их мировоззренческих принципов, определивших своеобразие их творчества (см., в частности: [25]). Значительно меныший интерес вызывало наличие в идейном наследии Кандинского и Шёнберга основных принципов, выходящих за пределы культуры модерна. Они долгое время не привлекали внимания по причине отсутствия соответствующей реальности и стали вызывать интерес лишь во второй половине двадцатого века.

Становящаяся реальностью культура постмодерна неожиданно высветила в теоретических построениях и художественной практике Кандинского и Шёнберга такие пласты смысла, которые первоначально рассматривались как нововведения, важные сами по себе, но имеющие значение исключительно для нетрадиционных теорий живописи и музыки соответственно, а также искусства в целом. Между тем при ближайшем рассмотрении обнаруживается, что интерес Кандинского к атональной музыке, а Шёнберга к абстрактной живописи имеет более глубокие причины, нежели определенная общность мировоззренческих установок, и более широкий контекст, нежели привычные отсылки к близости эстетических принципов и идеалов. В своих эстетике и философии искусства Кандинский и Шёнберг не только опередили развитие модерна, но и предвосхитили его переход в постмодерн.

Известно, что модернизация традиционных обществ всегда начинается с разрушения предшествующего общества, считающегося традиционным. В результате люди, в традиционных обществах занимавшие довольно жестко фиксированные места в своих социальных ячейках, теперь лишаются всех связей. Исходное состояние, получившееся в ито- ге разрушительной фазы модернизации, в высшей степени хаотично и проявляется в постоянной, неустранимой вражде (см. о процессах разрушения традиции в ходе модернизации: [7; $8 ; 13 ; 15 ; 26])$. Затем получившимся в результате массам необходимо было придать заранее предопределенные формы и устанавливать между ними структурные связи, также заранее спланированные, что и нашло свое отражение в концепции общественного договора.

Лишившись основания своего бытия в виде традиции после подготовительной работы модерна, и в теории, и на практике индивид может апеллировать только к самому себе, что, собственно, и превращает его в то особое основание, которое в западной философской традиции принято именовать субъектом. Из-за отсутствия других оснований субъект стремится опереться исключительно на самого себя в качестве cogito, считающегося отныне самодостаточным сущим и потому выступающим как наиболее прочное основание [9; 27]. В семиотическом плане это означает деконтекстуализацию, то есть изъятие из прежнего контекста. Новое объединение разрозненных, деконтекстуализированных, «абстрактных» частей целого - это решение задачи установления связи между единым и многим, частью и целым, которая стояла и перед традиционными обществами, но решалась там принципиально иначе, поскольку их внутренняя дифференциация была выражена очень слабо.

В исторической ретроспективе универсальным средством теоретического моделирования этой проблемы в европейской интеллектуальной традиции выступала метафизика, которая, независимо от того, в каких конкретных формах она представала, выполняла функцию нормативной порождающей структуры всех прочих структур, в том числе и структуры общественных отношений. Именно метафизика выступает как теоретическая модель взаимоотношений единого и много, целого и частей и, наконец, единичного, особенного и всеобщего, что свидетельствовало об углубляющейся социальной дифференциации. В этом отношении особое значение име- 
ют две основные характеристики метафизики, которые более или менее явно должны воспроизводиться и обнаруживаться во всех остальных структурах, включая и живопись, и музыку.

Первая принципиально важная черта метафизики достаточно давно выявлена и подробно описана. Метафизика в качестве онтотео-логии [3] выступает как «центрированная» система, то есть как иерархически организованная совокупность элементов, группирующихся вокруг некоторого выделенного элемента, выполняющего функцию центра. В сущности наличие такой структуры и означает, что метафизика представляет собой не только учение о сущем (онтологию), но и учение о высшем сущем (теологию). В результате в центрированных системах, нормативной моделью которых является метафизика, непосредственные взаимоотношения элементов между собой невозможны и они всегда опосредуются центром в качестве необходимого всеобщего эквивалента. Именно эта метафизическая структура воспроизводится во всех прочих формах «центризма»: таких, например, как фоно- и логоцентризм (см. подробнее: [17]).

На вторую принципиально важную черту метафизики, хотя она не менее существенна, обычно обращают значительно меньше внимания. Между тем метафизика вплоть до наступления эпохи постмодерна всегда рассматривалась не только как центрированная, но и как замкнутая система. Если события внутри системы имеют результатом явления и процессы, которые остаются внутри нее и, следовательно, должны считаться ее элементами, так что никакие новые элементы не могут быть добавлены, то система является замкнутой. На языке современной теории систем субстанциальность замкнутой системы можно также охарактеризовать, сказав, что имеется в виду такая подсистема, которая не находится в обратной связи со своим окружением [29, p. 356-361].

Понимание индивида в качестве самоутверждающегося субъекта, будучи основополагающим свойством модерна, существенно модифицирует метафизику, которая становится метафизикой субъективности. Ее необходимым элементом является требование надындивидуального, или, если восполь- зоваться кантовским термином, трансиендентального, субъекта. С одной стороны, он объединяет все эмпирические субъекты, а с другой - осуществляет свою синтезирующую деятельность, не возвышаясь над эмпирическими субъектами и не пребывая в неких недосягаемых сферах, а некоторым образом «присутствуя» в каждом из них (в кантовской философии - в качестве априорных форм, обеспечивающих всеобщность содержания чувственности и рассудка).

Именно метафизика субъективности получает наименование «диалектика» в смысле, который заметно отличается от смысла того, что понималось под «диалектикой» прежде. При этом гегелевская диалектика самим ее создателем понимается как спекулятивная (от лат. speculum - зеркало) потому, что противоположности «отсвечивают», то есть зеркально отражаются друг в друге [2, с. 383]. Этот процесс протекает в соответствии со схемой «тезис антитезис - синтез», в результате чего происходит схватывание взаимосвязи в качестве единства противоположностей.

Именно описанные принципы метафизики субъективности в полной мере реализовались и в живописи, и в музыке модерна, но именно от них в своем творчестве и в своих теоретических работах, предвосхищая постмодерн, отошли и Кандинский, и Шёнберг. Однако представляется правдоподобным предположение, что ведущую роль при этом играла не живопись, а музыка. Анализ теоретического и эпистолярного наследия Кандинского свидетельствует о том, что в своей концепции абстрактного искусства он вполне сознательно ориентировался на музыку вообще, прежде всего в связи с ее нерепрезентативностью.

Что касается позиции Шёнберга, то представляется достаточно убедительной точка зрения, основанная на анализе его теоретического и эпистолярного наследия, что он не искал в творчестве и теоретических работах Кандинского стимул и образец. Скорее, он нуждался в подтверждении правильности своих эстетических принципов и радикальных новшеств в теории музыки, а также универсальности тех изменений в искусстве, которые связывались с его деятельностью. Поэтому выявление концепции постмодерна в творчестве Кандинского становится значи- 
тельно менее сложной задачей, если оно соотносится с контекстом аналогичных процессов, нашедших свое выражение в концепции атональной музыки Шёнберга.

Не вдаваясь в технические подробности, отметим, что античная теория музыкального строя основывалась, как известно, на пифагорейской идее о том, что все (в первую очередь система отношений между звуками) подчиняется числу. Эффект совпадения или, точнее, полного слияния звука некоторой частоты и звука вдвое большей частоты (то есть совершенный консонанс) привел к открытию октавы - интервала между повторяющимися звуками. Поэтому октава является фундаментальным структурным элементом музыкального строя, который благодаря октавному удвоению может быть организован циклически. Лишь в результате открытия самого принципа цикличности - интервала $1: 2$, который, судя по всему, стал известен задолго до пифагореизма, появилась возможность организовывать музыкальный строй именно как замкнутую систему [22].

Благодаря принципу октавного удвоения, звуки, в определенном смысле оставаясь самими собою, могут быть перемещены из других октав в исходную октаву. Иначе говоря, все действия с ними вследствие совпадения звуков при октавном удвоении не должны приводить к появлению качественно новых элементов звукоряда как системы. Тогда структура октавы была бы инвариантной, а система октав - самоподобной. Правда, на самом деле, в музыке удвоение звуков, находящихся внутри данной октавы, не приводит к их точному повторению по образцу октавного удвоения. Цикличность, повторение как возвращение к исходному состоянию, не может быть совершенной, что выразительно проявляется в существовании так называемой пифагоровой коммы. Она указывает на то, что замыкание оказывается невозможным и вместо круга получается спираль.

Характерно, однако, что отказа от требования организации музыкального строя в качестве замкнутой системы вплоть до конца эпохи модерна не произошло. В результате оно сохраняется именно как не подлежащий обсуждению императив, что нашло выражение в концепции равномерной темперации как подчинении музыкального строя рациональным требованиям [28]. В результате выравнивания интервальных соотношений структура октавы, разделенная на двенадцать равных полутонов (хроматическая гамма), становится приблизительно инвариантной. Точно так же повторение после октавного удвоения становится почти совершенным, так что музыкальный строй моделируется все же как замкнутая система, хотя и с определенными оговорками.

В этом контексте формируется и концепция европейской тональности, из-за характерных особенностей которой ее даже приблизительные аналоги могут быть обнаружены далеко не во всех культурах. Эта концепция основана на представлении о том, что звуки разделяются на сильные (устойчивые) и слабые (неустойчивые), причем вторые испытывают более или менее легко ощущаемое слухом тяготение к первым. Тяготение в системе звуков проявляется как психологически достоверное ощущение напряжения от некоей незавершенности и необходимости такого перехода от одного звука к другому, который должен привести к желаемой завершенности. В общем смысле тональность является особой моделью возникновения напряжения и его снятия, возвращения в состояние покоя.

Главное в концепции новоевропейской тональности то, что для разделения звуков на сильные и слабые требуется некое подобие точки отсчета, то есть предельно устойчивый звук, обозначающий расслабление и покой. От него формируется звукоряд, относительно которого становится возможной система тяготений слабых звуков к сильным. Основной звук становится неподвижным тональным центром, тоникой, а совокупность отношений между звуками - ладом в качестве системы звуков, которая структурируется как иерархия функциональных звуковысотных отношений именно вследствие центрального положения тоники. Собственно новоевропейская тональность понимается как определенная высота звуков лада, которая обусловливается положением тоники на некоторой ступени звукоряда (см. подробнее: [4]). В структуре тональности, наряду с «центрацией», присутствует и цикличность. Действительно, гармоническое движение в аккордике, логика следования ак- 
кордов, строящихся на ступенях лада, также воспроизводит систему тяготений.

В этой связи необходимо подчеркнуть, что культура, в которой Шёнберг формировался как композитор, была буквально пронизана диалектическими моделями мысли, преимущественно идеями гегелевской диалектики [10, p. 46-51]. Именно против нее выступил Шёнберг, противопоставив ей свою концепцию атональной, двенадцатитоновой (додекафонической) музыки. По сути дела, с помощью музыкальных средств Шёнберг подверг пересмотру концепцию единства противоположностей в том виде, в котором она осмыслялась в европейской философии как метафизике субъективности. В результате отвергаются и идея иерархичности системы, и требование ее замкнутости. На языке теории музыки Шёнберг метафорически подверг критике гегелевское понятие «снятие» и само понятие системы (разумеется, замкнутой), хотя, вообще говоря, эта связь у него выражена довольно слабо и нуждается в теоретической реконструкции.

Нетрудно видеть, однако, куда ведет логика отказа от концепции иерархической замкнутой системы в качестве базовой концептуальной модели культуры модерна. Следуя этой логике, Шёнберг неизбежно должен был узаконить права диссонанса [11], и для него борьба противоположностей в мире звуков уже не может привести к их единству, как это имеет место в тональной музыке [21]. Очевидно, это обусловливается отказом Шёнберга от самого понятия тоники в качестве структурирующего центра. Звуки, лишившиеся абсолютной точки отсчета и не разделяющиеся на устойчивые и неустойчивые, в соответствии со специфической музыкальной «теорией относительности», должны считаться принципиально равноправными [16; 24].

Поэтому в основе атональной музыки лежит не диатоническая, а исключительно хроматическая гамма. Иными словами, в атональной музыке, неразрывно связанной с равномерной темперацией, используются все двенадцать звуков в октаве, причем под каждым звуком конкретной октавы подразумеваются все подобные звуки во всех октавах. Кроме того, постулируется еще и так называемая энгармоническая эквивалентность, в соответ- ствии с которой, к примеру, фа-диез и сольбемоль считаются, в отличие от классического понимания, не независимыми звуками, а одним и тем же звуком.

Таким образом, в двенадцатитоновой системе имеется всего двенадцать звуков и их значение является не абсолютным (в смысле принадлежности к определенной октаве), а относительным. Тем не менее использования только хроматической гаммы, отказа от концепции тоники, следования принципу энгармонической эквивалентности и ограничения числа звуков двенадцатью все же недостаточно для установления их полного равноправия. Для этого необходимо, чтобы в музыкальной композиции все звуки появлялись одинаковое число раз, что и вызвало к жизни понятие серии циклически организованной последовательности из всех двенадцати звуков хроматической гаммы, причем цикличность позволяет произвольно изменять начальный звук серии.

Серия выполняет функцию мелодии, но подчинена весьма специфическому правилу, согласно которому ни один звук не может появиться вторично до того, как прозвучат остальные звуки серии. С помощью различных преобразований высоты, длительности нот и многих других параметров звука, то есть достаточно формальных процедур, из основной серии могут быть получены производные, создание которых и становится основной задачей композитора. В итоге новоевропейская тональность была «децентрирована» - впрочем, насколько можно судить, без использования самого термина.

«Децентрация» Шёнбергом новоевропейской тональности имеет эвристическое значение для обнаружения и прослеживания параллелей того, что было сделано в творчестве и эстетике Кандинского. Нетрудно заметить, что в фундаментальной для Кандинского концепции единства всех видов искусства парадоксальным образом присутствует идея не только параллелизма музыки и живописи, но и главенства музыки. Именно живопись считается подражанием музыке, а не наоборот, и цвета, как постулируется, должны соответствовать звукам. Однако главное, что мешало осуществить в живописи те же структурные преобразования, что и в музыке, - репрезентативный характер живописи, и Кандинс- 
кий отказался от него, выдвинув концепцию беспредметной, такой же нерепрезентативной, как и музыка, абстрактной живописи.

Приравнивание живописи музыке потребовало в качестве первого шага отказа от основания репрезентативности живописи новоевропейской концепции перспективы. В живописи оба принципа метафизики в качестве нормативной «центрированной» замкнутой системы, охарактеризованные выше, наиболее последовательно реализуются именно в концепции перспективы. В этой связи необходимо обратить внимание на то, что перспектива в культуре модерна - это отнюдь не совокупность чисто технических приемов. В сущности это теоретические принципы и законы, позволяющие изображать предметы на плоскости с такими изменениями их размеров, пропорций и очертаний, чтобы при взгляде на плоскостные фигуры возникала иллюзия размещения того, что они изображают в реальном трехмерном пространстве. Поэтому так понимаемая перспектива служит целям иллюзионизма, то есть создания правдоподобной, хотя и иллюзорной визуальной репрезентации реальности.

Она должна вызывать у зрителя предельно достоверное ощущение подлинности, веры в то, что, оставаясь неподвижным и смотря на плоскую фигуру, он видит, как предмет выглядит в трехмерном пространстве, причем это не сопровождается чувством, что фактически он имеет дело с иллюзией [1]. В чисто теоретическом отношении принципы и законы перспективы довольно просты. Изобразить некоторую реальность значит привести точки трехмерного пространства в соответствии с точками некоторого двухмерного пространства, то есть плоскости. С позиций математической теории множеств это возможно, но затрагивает строение изображаемого объекта, то есть либо его непрерывность, либо взаимную однозначность отображаемых точек.

Известные принципы и законы перспективы позволяют воспроизвести на плоскости содержание пространства, но не его организацию (структуру). Поэтому перспектива воспроизводит лишь деформированное подобие действительности, а не ее точную копию [5; 14]. Использование законов перспективы предполагает не схематичное изображение отдель- ного предмета или отдельных предметов в их разрозненности, а уподобление изображения некоему «окну», открывающему зрителю вид целостного фрагмента действительности. Такое понимание предполагает, что человек (художник или зритель) в качестве конечного субъекта не только четко отделяет себя от окружающего его мира, но даже противостоит ему, при всей своей конечности играя роль его центра.

Между тем без дополнительных предположений образ мира в его перспективном видении открывался бы как неупорядоченная совокупность отдельных «точек зрения», соответствующих восприятию реальности многочисленными эмпирическими субъектами. Ограниченность их обзора в качестве проявления их принципиальной конечности доказывалась бы невозможностью смотреть на мир, кроме как через «окна» разных размеров. В концепции перспективы эта трудность преодолевается путем предположения, что зрительное восприятие пронизано некими структурами всеобщности точно так же, как того требует нормативная теоретическая модель новоевропейской субъективности.

В обоих случаях особым образом устанавливается связь между эмпирическим и неким надындивидуальным субъектами, что делает содержание зрительного восприятия, организованного по законам перспективы, всеобщим. Для этого место неподвижного наблюдателя (художника или зрителя) стягивается в точку, соответствующую расположению одного (обычно правого) глаза, и с этой точкой соотносятся все элементы изображаемой действительности. Получающаяся плоскостная картина оказывается подобной зрительному образу, возникающему на сетчатке глаза некоторого «наблюдателя вообще» в качестве надындивидуального субъекта.

Возможность существования такого «наблюдателя вообще» обусловливается определенными предположениями относительно свойств самого пространства, точками которого являются и положения субъектов перспективного видения. Для этого пространство должно быть не только евклидовым, непрерывным, изотропным, но прежде всего абсолютным в смысле механики Исаака Ньютона, то есть субстанциальным. Теоретическая 
модель пространства должна быть сконструирована таким образом, чтобы не допустить самодостаточности и, стало быть, привилегированного положения какого-либо отдельного наблюдателя, за исключением, конечно же, того субъекта, который своими особыми качествами и своим особым положением только и может задать абсолютную «точку зрения» и, стало быть, «центр» мира (для Ньютона это, разумеется, Бог).

В результате того, что пространство понимается в качестве «центрированной» системы, содержание перспективного видения может быть непрерывно перенесено в любую пространственную область без изменений, обусловленных локальными факторами. С одной стороны, именно вследствие своей инвариантности содержание зрительного восприятия приобретает форму всеобщности. Однако с другой стороны, очевидно, что отказ от концепции перспективы еще не делает живопись беспредметной. Скажем, рисунки маленьких детей не являются перспективными, но их нельзя назвать беспредметными.

В то же время ясно, что беспредметная живопись совсем не нуждается в перспективе в качестве средства создания иллюзии трехмерности изображаемых предметов уже потому, что этих предметов просто нет, и ей не нужно поддерживать эту иллюзию. У Кандинского отказ от репрезентативности проходил сложно и обусловливался многими причинами и влияниями - религиозными, научными, оккультистскими [6, p. 293-308], но они далеко не всегда указывали на главную цель - максимальное сближение живописи с музыкой. Между тем новоевропейская концепция перспективы обеспечивает в живописи тот «центризм», который пронизывает всю культуру модерна, присутствуя и в теории музыки в качестве постулата об особом положении тоники.

Поэтому отказ от концепции перспективы, которую приходится отвергнуть вместе с отрицанием императива репрезентативности живописи, устраняет все препятствия на пути к «децентрации» и, тем самым, к отрицанию иллюзионизма в качестве идеала того искусства, которое считается предметным. Существенно, однако, что отрицание философских принципов, на которые опирается концепция перспективы, затрагивает также другие ас- пекты живописи, прежде всего организацию цветов. Для Кандинского как теоретика абстрактной, беспредметной живописи музыка важна не только потому, что она действительно нерепрезентативна по своей природе, но и потому, что звук отождествляется им с духом. Дух для Кандинского - это «внутренний звук», присущий каждому объекту, так что весь мир для него предстает как звучащий [18, p. 59-64]. Это объясняет, почему музыке, а не живописи принадлежит главенствующая роль, так что живописец должен следовать именно ее принципам, одухотворяя цвет.

Вообще говоря, Кандинский был не единственным выразителем такой точки зрения (похожие взгляды были характерны также для Пита Мондриана). Однако задача установления параллелизма живописи и музыки путем отождествления цветов со звуками представляется довольно неопределенной и не слишком эвристичной, если этот параллелизм не конкретизируется. Кроме того, реконструкция логики такой конкретизации, которой следует Кандинский, такова, что главным оказывается не столько сам параллелизм цветов и звуков в качестве двух более или менее автономных совокупностей элементов. Особое значение имеют, скорее, системные характеристики самих этих совокупностей.

В этой связи нельзя не обратить внимания на то, что традиция организации цветов достаточно почтенна и подробно исследована на основе обширного материала $[12 ; 20]$. К сожалению, принципы этой организации, которые к тому же далеко не всегда были явно выражены, очень редко рассматривались в качестве предмета философского анализа. Между тем анализ истории становления этих принципов показывает, что они имеют целью «центрацию» цветов точно так же, как это было сделано с музыкальными звуками. Более того, само установление параллелизма структурной организации цветов и звуков является очень давней традицией [19; 23].

Именно в системных характеристиках предположительно - должно проявляться некое единообразие способов организации и цветов, и звуков. Кандинский подробно анализирует этот вопрос, предсказуемо обнаруживая у цветов аналоги тех тяготений, которые существуют в ладе между неустойчивыми и 
устойчивыми звуками [18, p. 66-112]. Однако в итоге Кандинский, точно так же как Шёнберг, отказался от понятия тоники, отвергает теоретическую модель «центрированной» системы в качестве образца для организации цветов (цветовых звуков).

При этом Кандинский прямо ссылается и на атональную музыку Шёнберга, и на его понимание каждого звука в качестве свободного [18, p. 114-130] (если, конечно, не принимать во внимание ограничивающие свободу законы организации звуков в серию). Приписывая каждому отдельному цвету единственное в своем роде эмоциональное и духовное воздействие, Кандинский настаивает на такой организации цветов, в которой, как и в атональной музыке, главным является разлад, неснятое противоречие, аналогичное диссонансу в атональной музыке. Соответственно, композиция, согласно Кандинскому, может покоиться на такой антилогике сочетания красок, которые прежде считались дисгармоничными.

Это такая «гармония противоположностей», в которой невозможность их сочетать сохраняется, но их объединение считается аккордом самостоятельно существующих красочных и рисуночных форм [18, p. 109]. Это указывает на то, что понимание гармонии в абстрактной живописи с удивительной точностью соответствует постмодернистскому пониманию гармонии в атональной музыке. Более того, точно так же как и атональная музыка, оно предвосхищает отрицание той концепции целостности, которая является конститутивной для философии и идеологии модерна. Главными следствиями этого отрицания являются изменения статуса самоутверждающегося субъекта модерна. Одновременно перестают работать механизмы, порождающие и поддерживающие убежденность модерна в своей универсальности и вечности, которые, как казалось, следуют из его единственных в своем роде метафизических оснований.

\section{СПИСОК ЛИТЕРАТУРЫ}

1. Флоренский, П. А. Обратная перспектива / П. А. Флоренский // Флоренский, П. А. Сочинения. В 4 т. Т. 3 / сост. игумена Андроника (А. С. Трубачева), П. В. Флоренского, М. С. Трубачевой ; ред. игумен Андроник (А. С. Трубачев). - М. : Мысль, 2000. - С. 46-103.

2. Хайдеггер, М. Гегель и греки / М. Хайдеггер // Время и бытие / М. Хайдеггер ; пер. с нем. В. В. Бибихина. - М. : Республика, 1993. C. $381-390$.

3. Хайдеггер, М. Онто-теологическое строение метафизики / М. Хайдеггер // Тождество и различие / М. Хайдеггер; пер. с нем. А. Денежкина, науч. ред. О. Никифоров. - М. : Гнозис : Логос, 1997. - С. 29-59.

4. Agmon, E. The Languages of Western Tonality / E. Agmon. - Berlin ; Heidelberg : Springer, 2013. - XXVIII, $280 \mathrm{p}$.

5. Andersen, K. The Geometry of an Art: The History of the Mathematical Theory of Perspective from Alberti to Monge / K. Andersen. - N. Y. : Springer Science + Business Media, 2007.XXXVII, $812 \mathrm{p}$.

6. Barash, M. Modern Theories of Art. Vol. 2: From Impressionism to Kandinsky / M. Barash. - N. Y. ; L. : New York University Press, 1998. - X, 390 p.

7. Berman, M. All That is Solid Melts into Air: The Experience of Modernity / M. Berman. - N. Y. : Penguin Books, 1988. -233 p.

8. Bertens, H. The Idea of the Postmodern: A History /H. Bertens. - L. ; N. Y. : Routledge, 1995.IX, $280 \mathrm{p}$.

9. Blumenberg, H. Säkularisierung und Selbstbehauptung (Erweiterte und überarbeitete Neuausgabe von "Die Legitimität der Neuzeit", erster und zweiter Teil) / H. Blumenberg. - Frankfurt a.M. : Suhrkamp, 1974. $-294 \mathrm{p}$.

10. Cherlin, M. Schoenberg's Musical Imagination / M. Cherlin. - Cambridge : Cambridge University Press, 2007. - XVI, $397 \mathrm{p}$.

11. Constructive Dissonance: Arnold Schoenberg and the Transformations of TwentiethCentury Culture / ed. by J. Brand, C. Hailey.Berkeley; Los Angeles ; L. : University of California Press, 1997. - XVIII, $232 \mathrm{p}$.

12. Crone, R. A. History of Colors: The Evolution of Theories of Light and Color / R. A. Crone. Dordrecht ; Boston ; L. : Kluwer Academic Publishers, 2000. $-282 \mathrm{p}$.

13. Delanty, G. Modernity and Postmodernity: Knowledge, Power and the Self / G. Delanty. - L. ; Thousand Oaks ; New Delhi : SAGE Publications, 2000. - XIV, $194 \mathrm{p}$.

14. Edgerton, S. Y. The Mirror, the Window, and the Telescope: How Renaissance Linear Perspective Changed Our Vision of the Universe / S. Y. Edgerton. - Ithaca : Cornell University Press, 2009. - XVIII, $199 \mathrm{p}$.

15. Formations of Modernity / ed. by S. Hall, B. Gieben. - Cambridge : Polity Press, 1995. - IX, 342 p. 
16. Forte, A. The Structure of Atonal Music / A. Forte. - New Haven ; L. : Yale University Press, 1973. - X, 224 p.

17. Goux, J.-J. Symbolic Economies: After Marx and Freud / J.-J. Goux ; trans. by J. C. Gage. - Ithaca : Cornell University Press, 1990. - VII, 257 p.

18. Kandinsky, W. Über das Geistige in der Kunst. 4. Aufl., mit einer Einführung von Max Bill / W. Kandinsky. - Bern ; Bümplitz : Benteli-Verlag, 1952. - $144 \mathrm{p}$.

19. Kuehni, R. G. Color Ordered: A Survey of Color Order Systems from Antiquity to the Present / R. G. Kuehni, A. Schwarz. - N. Y. : Oxford University Press, 2008. - XIV, 391 p.

20. Kuehni, R. G. Color Space and Its Divisions: Color Order from Antiquity to the Present / R. G. Kuehni. - Hoboken : John Wiley \& Sons, 2003. $\mathrm{XIV}, 408 \mathrm{p}$.

21. Leibowitz, R. Schoenberg and His School: The Contemporary Stage of the Language of Music / R. Leibowitz ; trans. by D. Newlin. - N. Y. : Da Capo, 1979. - XXVI, $305 \mathrm{p}$.

22. McClain, E. G. The Myth of Invariance: The Origin of the Gods, Mathematics and Music From the Rg Veda to Plato / E. G. McClain. - York-Beach : Nicolas-Hays, Inc., 1984. - XIX, 216 p.

23. Riley, C. A. II. Color Codes: Modern Theories of Color in Philosophy, Painting and Architecture, Literature, Music, and Psychology / C. A. Riley II. - Hanover ; L. : University Press of New England, 1995. - XI, 351 p.

24. Schoenberg, A. Style and Idea: Selected Writings of Arnold Schoenberg / A. Schoenberg ; trans. by L. Blackn ; ed. by L. Stein. - Berkeley : University of California Press, 1985. - 559 p.

25. Schönberg and Kandinsky: An Historic Encounter/ ed. by K. Boehmer. - N. Y.; L. : Routledge, 2011. - XVIII, $224 \mathrm{p}$.

26. Theories of Modernity and Postmoderniy / ed. by B. S. Turner. - L.; Thousand Oaks ; New Delhi : SAGE Publications, 1995. - IX, 183 p.

27. Venn, C. Occidentalism: Modernity and Subjectivity / C. Venn. - L. ; Thousand Oaks ; New Delhi : SAGE Publications, 2000. - VIII, 256 p.

28. Weber, M. Die rationalen und soziologischen Grundlagen der Musik. Mit einer Einleitung von Prof. Dr. T. Kroyer / M. Weber. - München : Drei Masken Verlag, 1921.-VIII, 94 p.

29. Wilden, A. System and Structure: Essays in Communication and Exchange / A. Wilden. - L. ; N. Y. : Tavistock Publications, 1980. - LXIV, 592 p.

\section{REFERENCES}

1. Florenskiy P.A. Obratnaya perspektiva [The Reverse Perspective]. Florenskiy P.A. Sochineniya.
V 4 t. T. 3 [Selected Writings. In 4 vols. Vol. 3]. Moscow, Mysl Publ., 2000, pp. 46-103.

2. Haidegger M. Gegel i greki [Hegel and the Greeks]. Haidegger M. Vremya i bytie [Time and Being]. Moscow, Respublika Publ., 1993, pp. 381-390.

3. Haidegger M. Onto-teologicheskoe stroenie metafiziki [The Onto-theological Constitution of Metaphysics]. Heidegger M. Tozhdestvo i razlichie [Identity and Difference]. Moscow, Gnozis Publ.; Logos Publ., 1997, pp. 29-59.

4. Agmon E. The Languages of Western Tonality. Berlin; Heidelberg, Springer, 2013. XXVIII, $280 \mathrm{p}$.

5. Andersen K. The Geometry of an Art: The History of the Mathematical Theory of Perspective from Alberti to Monge. New York, Springer Science+Business Media, 2007. XXXVII, 812 p.

6. Barash M. Modern Theories of Art. Vol. 2: From Impressionism to Kandinsky. New York; London, New York University Press, 1998. X, 390 p.

7. Berman M. All That is Solid Melts into Air: The Experience of Modernity. New York, Penguin Books, 1988. $233 \mathrm{p}$.

8. Bertens H. The Idea of the Postmodern: A History. London; NewYork, Routledge, 1995. IX, 280 p.

9. Blumenberg H. Säkularisierung und Selbstbehauptung (Erweiterte und überarbeitete Neuausgabe von "Die Legitimität der Neuzeit", erster und zweiter Teil). Frankfurt a.M., Suhrkamp, 1974. $294 \mathrm{p}$.

10. Cherlin M. Schoenberg's Musical Imagination. Cambridge, Cambridge University Press, 2007. XVI, 397 p.

11. Brand J., Hailey, C., eds. Constructive Dissonance: Arnold Schoenberg and the Transformations of Twentieth-Century Culture. Berkeley; Los Angeles; London, University of California Press, 1997. XVIII, 232 p.

12. Crone R.A. History of Colors: The Evolution of Theories of Light and Color. Dordrecht; Boston; London, Kluwer Academic Publishers, 2000. 282 p.

13. Delanty G. Modernity and Postmodernity: Knowledge, Power and the Self. London; Thousand Oaks; New Delhi, SAGE Publications, 2000. XIV, 194 p.

14. Edgerton S.Y. The Mirror, the Window, and the Telescope: How Renaissance Linear Perspective Changed Our Vision of the Universe. Ithaca, Cornell University Press, 2009. XVIII, 199 p.

15. Hall S., Gieben B., eds. Formations of Modernity. Cambridge, Polity Press, 1995. IX, 342 p.

16. Forte A. The Structure of Atonal Music. New Haven; London, Yale University Press, 1973. X, 224 p.

17. Goux J.-J. Symbolic Economies: After Marx and Freud. Ithaca, Cornell University Press, 1990. VII, 257 p. 
18. Kandinsky W. Über das Geistige in der Kunst. Bern; Bümplitz, Benteli-Verlag, 1952. 144 p.

19. Kuehni R.G., Schwarz A. Color Ordered: A Survey of Color Order Systems from Antiquity to the Present. New York, Oxford University Press, 2008. XIV, $391 \mathrm{p}$.

20. Kuehni R.G. Color Space and Its Divisions: Color Order from Antiquity to the Present. Hoboken, John Wiley \& Sons, 2003. XIV, 408 p.

21. Leibowitz R. Schoenberg and His School: The Contemporary Stage of the Language of Music. New York, Da Capo, 1979. XXVI, 305 p.

22. McClain E.G. The Myth of Invariance: The Origin of the Gods, Mathematics and Music from the R. Veda to Plato. York-Beach, Nicolas-Hays, Inc., 1984. XIX, 216 p.

23. Riley C.A. II. Color Codes: Modern Theories of Color in Philosophy, Painting and Architecture, Literature, Music, and Psychology. Hanover; London, University Press of New England, 1995. XI, 351 p.
24. Schoenberg A. Style and Idea: Selected Writings of Arnold Schoenberg. Berkeley, University of California Press, 1985. 559 p.

25. Boehmer K., ed. Schönberg and Kandinsky: An Historic Encounter. New York; London, Routledge, 2011. XVIII, 224 p.

26. Turner B.S., ed. Theories of Modernity and Postmoderniy. London; Thousand Oaks; New Delhi, SAGE Publications, 1995. IX, 183 p.

27. Venn C. Occidentalism: Modernity and Subjectivity. London; Thousand Oaks; New Delhi, SAGE Publications, 2000. VIII, 256 p.

28. Weber M. Die rationalen und soziologischen Grundlagen der Musik [The Rational and Social Foundations of Music]. München, Drei Masken Verlag, 1921. VIII, $94 \mathrm{p}$.

29. Wilden A. System and Structure: Essays in Communication and Exchange. London; New York, Tavistock Publications, 1980. LXIV, 592 p.

\section{Information about the Author}

Aleksandr I. Pigalev, Doctor of Sciences (Philosophy), Professor of Department of Philosophy, Volgograd State University, Prosp. Universitetsky, 100, 400062 Volgograd, Russian Federation, pigalev@volsu.ru, socphil@volsu.ru.

\section{Информация об авторе}

Александр Иванович Пигалев, доктор философских наук, профессор кафедры философии, Волгоградский государственный университет, просп. Университетский, 100, 400062 г. Волгоград, Российская Федерация, pigalev@volsu.ru, socphil@volsu.ru. 